\title{
Interim Guidance: 4-Month Rifapentine-Moxifloxacin Regimen for the Treatment of Drug-Susceptible Pulmonary Tuberculosis — United States, 2022
}

\author{
Wendy Carr, $\mathrm{PhD}^{1}$; Ekaterina Kurbatova, $\mathrm{MD}^{1}$; Angela Starks, $\mathrm{PhD}^{1}$; Neela Goswami, $\mathrm{MD}^{1}$; Leeanna Allen, $\mathrm{MPH}^{1}$; Carla Winston, $\mathrm{PhD}^{1}$
}

\section{Summary}

On May 5, 2021, CDC's Tuberculosis Trials Consortium and the National Institutes of Health (NIH)-sponsored AIDS Clinical Trials Group (ACTG) published results from a randomized controlled trial indicating that a 4-month regimen containing rifapentine (RPT), moxifloxacin (MOX), isoniazid (INH), and pyrazinamide (PZA) was as effective as the standard 6-month regimen for tuberculosis (TB) treatment (1). On the basis of these findings, CDC recommends the 4-month regimen as a treatment option for U.S. patients aged $\geq 12$ years with drug-susceptible pulmonary TB and provides implementation considerations for this treatment regimen.

\section{Background}

Standard treatment for culture-positive TB requires $\geq 6$ months of antibiotics (2). Shorter, effective TB treatments could enable more rapid cure and improve patient quality of life. Sponsored by CDC and conducted in collaboration with the NIH-sponsored ACTG, Study 31/A5349 (https://clinicaltrials.gov/ct2/show/NCT02410772) was an international, open label, phase 3 noninferiority clinical trial that randomized 2,516 participants at 34 clinical sites in 13 countries. The trial confirmed that a 4-month daily treatment regimen containing high-dose RPT and MOX, as well as INH and PZA, is as effective as (noninferior to) the standard daily 6-month regimen in curing drug-susceptible TB (1).

\section{Methods}

CDC developed this interim guidance, based on evidence from Study 31/A5349, preclinical and animal evidence, previous clinical trial findings, pharmacokinetic and pharmacodynamic modeling $(1,3-6)$, and CDC expert opinion regarding considerations for implementation of the new 4-month daily treatment regimen in the United States. A systematic review framework was not applicable because this regimen has not been compared in other studies. A CDC writing group reviewed the evidence and drafted guidance for comments from external TB subject matter experts and for presentation for public comment. Comments were addressed by developing content to be published at https://www.cdc.gov/tb/topic/ treatment/tbdisease.htm.

\section{INSIDE}

290 Use of Ebola Vaccine: Expansion of Recommendations of the Advisory Committee on Immunization Practices To Include Two Additional Populations — United States, 2021

293 Antigen Test Positivity After COVID-19 Isolation Yukon-Kuskokwim Delta Region, Alaska, JanuaryFebruary 2022

299 Results from a Test-to-Release from Isolation Strategy Among Fully Vaccinated National Football League Players and Staff Members with COVID-19 - United States, December 14-19, 2021

306 Changes in Suicide Rates - United States, 2019 and 2020

313 Pediatric Emergency Department Visits Before and During the COVID-19 Pandemic - United States, January 2019-January 2022

319 Pediatric Emergency Department Visits Associated with Mental Health Conditions Before and During the COVID-19 Pandemic - United States, January 2019-January 2022

326 QuickStats

Continuing Education examination available at https://www.cdc.gov/mmwr/mmwr_continuingEducation.html

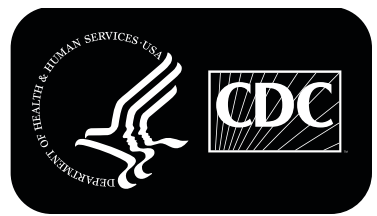

U.S. Department of Health and Human Services Centers for Disease Control and Prevention 


\section{Rationale and Evidence}

Clinical practice guidelines for treatment of drug-susceptible TB in the United States were published in 2016 (2). This interim guidance updates 2016 guidelines by recommending and providing implementation considerations for a novel 4-month daily treatment regimen, based on high-dose daily RPT with MOX, INH, and PZA (1) as a treatment option for U.S. patients aged $\geq 12$ years with drug-susceptible pulmonary TB. The regimen is intended for administration in settings where mycobacterial cultures, molecular and phenotypic drug susceptibility testing (DST), radiographic studies and other diagnostic tools, infrastructure for adverse event monitoring, patient-centered clinical care, and coordination with public health for case management are available.

\section{Recommendation for Use of the 4-month Rifapentine-Moxifloxacin Regimen}

CDC recommends the 4-month RPT-MOX regimen for treating patients aged $\geq 12$ years with body weight $\geq 40 \mathrm{~kg}$ with pulmonary TB caused by organisms that are not known or suspected to be drug-resistant and who have no contraindications to this regimen. The 4-month daily treatment regimen consists of an intensive phase composed of 8 weeks of daily treatment with RPT, MOX, INH, and PZA, followed by a continuation phase of 9 weeks of daily treatment with RPT, MOX, and INH (Table 1). Anti-TB drugs should be administered once daily with food, 7 days per week, for a total of 119 treatment doses; similar to the standard 6-month regimen, at least 5 of 7 weekly doses should be administered under direct observation (2). The 4-month regimen can be used in persons with an HIV infection who have CD 4 counts $\geq 100$ cells $/ \mu \mathrm{L}$ and are receiving or planning to initiate efavirenz as part of their antiretroviral therapy (ART) regimen in the absence of any other known drug-drug interactions between antituberculosis and antiretroviral medications.

Considerations. The 4-month daily treatment regimen was not studied in, and CDC does not recommend this regimen for, the following patient groups: body weight $<40 \mathrm{~kg}$; age $<12$ years; pregnant or breastfeeding; most types of suspected or documented extrapulmonary TB infection (see exceptions below); history of prolonged QT syndrome or concurrent use of one or more QT-prolonging medications (in addition to MOX); patients receiving medications with known clinically relevant drug-drug interactions with RPT, MOX, INH, or PZA; or patients infected with a baseline Mycobacterium tuberculosis isolate known or suspected to be resistant to INH, PZA, rifampin (RIF), or fluoroquinolones.

The 4-month daily treatment regimen was not studied in, and CDC recommends that clinical consultation be obtained to determine if this regimen is an acceptable treatment option for, patient groups with increased risk for $M$. tuberculosis resistance to any drug in the regimen, including persons who received $>5$ doses of treatment directed against TB in the preceding 6 months, who received $>5$ doses of latent tuberculosis

The MMWR series of publications is published by the Center for Surveillance, Epidemiology, and Laboratory Services, Centers for Disease Control and Prevention (CDC), U.S. Department of Health and Human Services, Atlanta, GA 30329-4027.

Suggested citation: [Author names; first three, then et al., if more than six.] [Report title]. MMWR Morb Mortal Wkly Rep 2022;71:[inclusive page numbers].

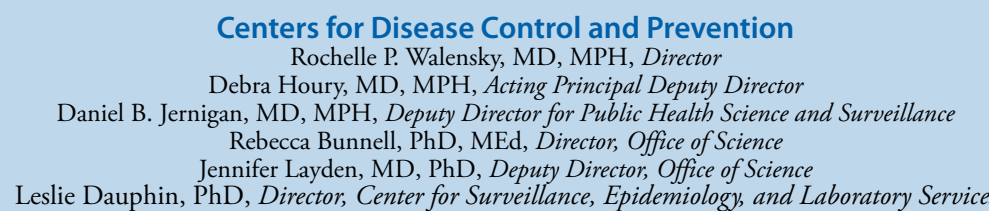

MMWR Editorial and Production Staff (Weekly)

Charlotte K. Kent, $\mathrm{PhD}$, MPH, Editor in Chief Jacqueline Gindler, MD, Editor

Paul Z. Siegel, MD, MPH, Associate Editor

Mary Dott, MD, MPH, Online Editor Terisa F. Rutledge, Managing Editor

Teresa M. Hood, MS, Lead Technical Writer-Editor

Leigh Berdon, Glenn Damon, Soumya Dunworth, PhD

Tiana Garrett-Cherry, PhD, MPH, Srila Sen, MA, Stacy Simon, MA, Morgan Thompson,

Technical Writer-Editors

Matthew L. Boulton, MD, MPH

Carolyn Brooks, ScD, MA

Jay C. Butler, MD

Virginia A. Caine, MD

Jonathan E. Fielding, MD, MPH, MBA

David W. Fleming, MD
Martha F. Boyd, Lead Visual Information Specialist Alexander J. Gottardy, Maureen A. Leahy,

Julia C. Martinroe, Stephen R. Spriggs, Tong Yang, Visual Information Specialists

Quang M. Doan, MBA, Phyllis H. King,

Terraye M. Starr, Moua Yang,

Information Technology Specialists
Ian Branam, MA,

Acting Lead Health Communication Specialist Shelton Bartley, MPH, Leslie Hamlin,

Lowery Johnson, Amanda Ray,

Health Communication Specialists Will Yang, MA,

Visual Information Specialist
MMWR Editorial Board

Timothy F. Jones, MD, Chairman

William E. Halperin, MD, DrPH, MPH

Jewel Mullen, MD, MPH, MPA

Jeff Niederdeppe, $\mathrm{PhD}$

Celeste Philip, MD, MPH

Patricia Quinlisk, MD, MPH

Patrick L. Remington, MD, MPH
Carlos Roig, MS, MA

William Schaffner, MD

Nathaniel Smith, MD, MPH

Morgan Bobb Swanson, BS

Abbigail Tumpey, MPH 
Morbidity and Mortality Weekly Report

TABLE 1. Dosing recommendation for a 4-month rifapentine-moxifloxacin regimen for patients aged $\geq 12$ years with pulmonary tuberculosis caused by drug-susceptible organisms - United States, 2022

\begin{tabular}{|c|c|c|c|c|c|}
\hline Medication* & Body weight, kg & Dose & Intensive phase & Continuation phase & Total doses \\
\hline Rifapentine & $\geq 40$ & $1,200 \mathrm{mg}$ & \multirow{6}{*}{7 days/wk for 56 doses ( 8 wks) } & 7 days/wk for 63 doses (9 wks) & \multirow{6}{*}{119} \\
\hline Moxifloxacin & $\geq 40$ & $400 \mathrm{mg}$ & & & \\
\hline Isoniazid $^{\dagger}$ & $\geq 40$ & $300 \mathrm{mg}$ & & \multirow{4}{*}{ NA } & \\
\hline \multirow[t]{3}{*}{ Pyrazinamide } & $40-<55$ & $1,000 \mathrm{mg}$ & & & \\
\hline & $\geq 55-75$ & $1,500 \mathrm{mg}$ & & & \\
\hline & $>75 \mathrm{~kg}$ & $2,000 \mathrm{mg}$ & & & \\
\hline
\end{tabular}

Abbreviation: NA = not applicable.

* Medications should be administered with food.

† Pyridoxine (vitamin B6), 25-50 mg/day, should be given with isoniazid to all patients.

infection treatment in the preceding 6 months, or who received $>5$ doses of treatment with any one or more of the following drugs for any reason (e.g., urinary tract infection or pneumonia) in the preceding 30 days: INH, RIF, rifabutin, RPT, PZA, or any fluoroquinolone. Other patient groups for whom clinical consultation is recommended include those with serum or plasma alanine aminotransferase or aspartate aminotransferase $>3$ times the upper limit of normal or total bilirubin $>2.5$ times the upper limit of normal, or with preexisting advanced liver disease; renal insufficiency or end-stage renal disease, or with serum or plasma creatinine level $>2$ times the upper limit of normal; plasma potassium level $<3.5 \mathrm{mEq} / \mathrm{L}$; who have types of extrapulmonary TB that are likely to be paucibacillary, not pose a substantial risk for death or disability, and not require prolonged treatment (i.e., pleural or lymph node TB); or for whom a specimen was unable to be submitted for any M. tuberculosis resistance testing before initiating treatment.

The 4-month daily treatment regimen was not studied in patients with a negative sputum culture, but who in the judgment of the clinician likely represent paucibacillary or low mycobacterial burden pulmonary TB disease. A 4-month regimen for smear-negative, culture-negative, noncavitary $\mathrm{TB}$ exists in the 2016 CDC guidelines (2), and CDC recommends that the 4-month RPT-MOX regimen may also be used unless patients are in one of the nonrecommended patient groups listed above.

Baseline and follow-up evaluations. Microbiology, laboratory, and clinical assessments are recommended before starting and during treatment with the 4-month daily regimen (Table 2). A respiratory specimen for acid-fast bacilli smear microscopy and culture should be obtained at baseline and at monthly intervals during treatment until two consecutive specimens are negative on culture. Baseline molecular drugsusceptibility testing for rapid identification of mutations associated with resistance to at least INH, PZA, RIF, and fluoroquinolones is advisable. Phenotypic DST should follow with a panel to include at least RIF (as surrogate for RPT), $\mathrm{INH}, \mathrm{PZA}$, and MOX as the preferred fluoroquinolone. CDC's

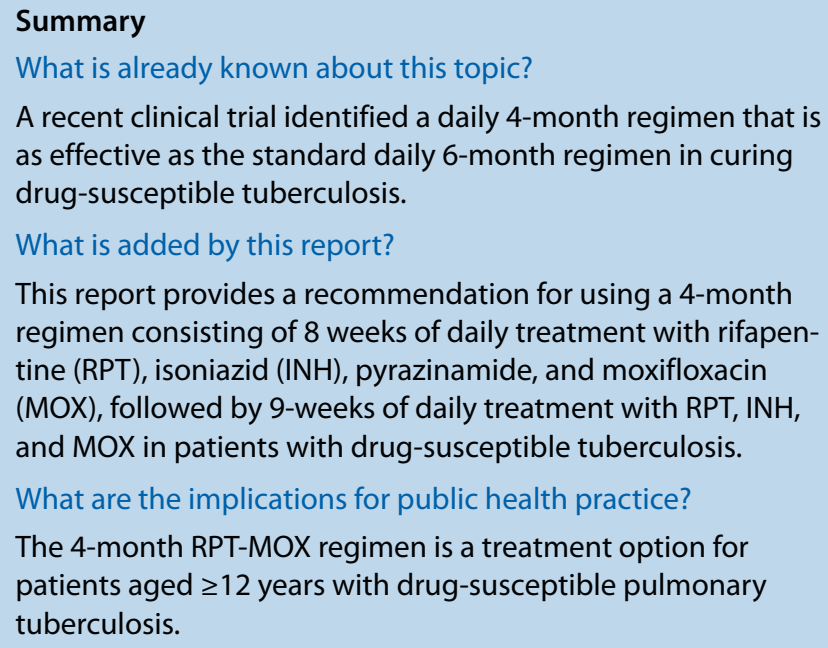

Tuberculosis Elimination Laboratory (TBLab@cdc.gov) can assist identifying laboratories to perform this testing for $\mathrm{TB}$ programs that intend to implement the 4-month daily treatment regimen.

Duration and definition of completion of therapy. The 4-month daily treatment regimen is considered complete based on the total number of doses taken (119). Recommended treatment duration is independent of any cavitation on baseline chest radiograph. Intensive phase doses (56) should be administered within 70 days from treatment initiation, and continuation phase doses (63) should be administered within 84 days from intensive phase completion, so that the regimen is completed within 5 months. If these targets are not met, the patient should be considered to have interrupted therapy and be managed as described in TB treatment guidelines (2). Confirmation of continued susceptibility to all drugs in the 4-month daily treatment regimen is required before restarting this regimen.

Poor treatment response and treatment failure or discontinuation. Patients with any positive culture at completion of 2 months of therapy, with or without ongoing symptoms, should be carefully evaluated to identify the cause of delayed 
TABLE 2. Baseline and follow-up evaluations for patients treated with a 4-month rifapentine-moxifloxacin regimen - United States, 2022*

\begin{tabular}{|c|c|c|c|c|c|}
\hline Evaluation & Baseline & Week 4 & $\begin{array}{c}\text { Week } 8 \\
\text { (end of intensive phase) }\end{array}$ & Week 12 & $\begin{array}{c}\text { Week } 17 \\
\text { (end of treatment) }\end{array}$ \\
\hline \multicolumn{6}{|l|}{ Microbiology } \\
\hline Sputum for rapid molecular test ${ }^{\dagger}$ & $\mathrm{Y}$ & NA & NA & NA & NA \\
\hline Sputum for AFB smear and culture ${ }^{\S}$ & Y & Y & Y & $Y^{\Uparrow}$ & $Y^{\natural}$ \\
\hline Drug susceptibility testing ${ }^{* *}$ & Y & NA & $Y^{\uparrow}$ & NA & NA \\
\hline \multicolumn{6}{|l|}{ Imaging } \\
\hline Chest radiograph ${ }^{\dagger \dagger}$ & Y & NA & $Y^{\uparrow}$ & NA & $Y^{\natural}$ \\
\hline \multicolumn{6}{|l|}{ Clinical assessment } \\
\hline Weight ${ }^{\S}$ & Y & Y & Y & Y & Y \\
\hline Symptoms, adverse events, and adherence ${ }^{\text {ๆी }}$ & Y & Y & Y & Y & Y \\
\hline \multicolumn{6}{|l|}{ Laboratory testing } \\
\hline ALT, AST, bilirubin, and alkaline phosphate ${ }^{* * *}$ & Y & $Y^{\natural}$ & $Y^{\natural}$ & $Y^{\natural}$ & $Y^{\natural}$ \\
\hline Platelet count & Y & $Y^{\natural}$ & $Y^{\pi}$ & $Y^{\natural}$ & $Y^{n}$ \\
\hline Creatinine & Y & $Y^{\natural}$ & $Y^{\natural}$ & $Y^{\pi}$ & $Y^{n}$ \\
\hline Potassium, calcium, and magnesium ${ }^{\dagger+\dagger}$ & Y & $Y^{\natural}$ & $Y^{\pi}$ & $Y^{\pi}$ & $Y^{n}$ \\
\hline HIV & Y & NA & NA & NA & NA \\
\hline CD4 count and HIV RNA load (if HIV infection) ${ }^{\S \S \S}$ & $Y^{\natural}$ & NA & NA & NA & NA \\
\hline Hepatitis B and C screen ${ }^{\text {१ी }}$ & $Y^{\natural}$ & NA & NA & NA & NA \\
\hline Diabetes screen ${ }^{* * * *}$ & $Y^{\natural}$ & NA & NA & NA & NA \\
\hline Pregnancy testing for persons who might become pregnant ${ }^{t+t \dagger}$ & $\mathrm{Y}$ & NA & NA & NA & NA \\
\hline
\end{tabular}

Abbreviations: $\mathrm{AFB}$ = acid-fast bacilli; $\mathrm{ALT}=$ alanine aminotransferase; $\mathrm{AST}=$ aspartate aminotransferase; $\mathrm{DST}=$ drug susceptibility testing; INH = isoniazid; $\mathrm{MOX}=$ moxifloxacin; $\mathrm{NA}=$ not applicable; $\mathrm{PZA}=$ pyrazinamide; $\mathrm{RIF}=$ rifampin; $\mathrm{RPT}=$ rifapentine; $\mathrm{Y}=$ yes .

* Regimen consists of RPT, MOX, INH, and PZA.

${ }^{\dagger}$ At least one baseline specimen is advised to be tested using a rapid molecular test for susceptibility to INH, PZA, RIF, and fluoroquinolones.

$\S$ Sputa for AFB smear and culture should be obtained at baseline, then monthly until two consecutive specimens are AFB smear-and culture-negative.

I These activities are optional or contingent on other information.

** Drug susceptibility at least for INH, RIF, PZA, and fluoroquinolones (preferred fluoroquinolone is MOX) should be obtained. Drug susceptibility testing (rapid molecular preferred) should be repeated if patient's culture remains positive after completing 2 months ( 8 weeks) of treatment.

${ }^{+\dagger}$ Chest radiograph should be obtained at baseline for all patients and at month 2 if baseline cultures are negative. End-of-treatment chest radiograph is optional. Electrocardiogram is not routinely recommended for all patients; electrocardiogram should be done if clinically indicated.

$\S \S$ Weight should be monitored monthly to assess response to treatment; adjust PZA dose if needed.

११ Adherence should be assessed, improvement in tuberculosis symptoms (e.g., cough, fever, fatigue, or night sweats) monitored, and development of medication adverse effects (e.g., jaundice, dark urine, nausea, vomiting, abdominal pain, diarrhea, anorexia, dizziness, seizures, fever, rash, malaise, neuropathy, arthralgias, tendinopathy, heart palpitations, irregular heartbeat, weakness, or syncope) evaluated.

*** Liver function tests only at baseline unless abnormalities at baseline, symptoms consistent with hepatotoxicity develop, or for patients who chronically consume alcohol, take other potentially hepatotoxic medications, or have viral hepatitis or history of liver disease, HIV infection, or previous drug-induced liver injury.

$\mathrm{tt+}^{+\dagger}$ Further monitoring if baseline abnormalities or clinically indicated.

$\S \S$ HIV testing in all patients; CD4 lymphocyte count and HIV RNA load testing if HIV infection.

११ๆ Hepatitis screening for all patients in accordance with CDC guidelines. Patients with hepatitis B or C risk factors or elevated baseline liver function tests should be tested for these viruses. https://www.cdc.gov/mmwr/volumes/69/rr/rr6902a1.htm

**** Fasting glucose or hemoglobin A1c for patients with risk factors for diabetes according to the American Diabetes Association, including age $>45$ years; body mass index $>25 \mathrm{~kg} / \mathrm{m}^{2}$; first-degree relative with diabetes; and race/ethnicity of African American, Asian, Hispanic, American Indian or Alaska Native, or Native Hawaiian or other Pacific Islander. For patients with diabetes, glucose monitoring is indicated. https://professional.diabetes.org/content-page/ practice-guidelines-resources

t+t+ Persons who can become pregnant should be advised to use a barrier contraceptive method, nonhormonal intrauterine device, or abstain from heterosexual intercourse during treatment.

response (2). Mycobacterial isolates obtained after 2 months should be sent to a reference laboratory for DST. If drug resistance to INH, RIF, PZA, or any fluoroquinolone is detected by any testing method (i.e., phenotypic or molecular) in baseline or follow-up specimens, the 4-month regimen should be stopped, and patients should be started on an appropriate treatment regimen that accounts for the identified drugresistance pattern (7). Patients who become pregnant while on treatment should receive clinical consultation regarding whether to stop the 4-month daily treatment regimen and be treated with an alternative regimen that is considered safer for pregnant persons (2).

\section{Discussion}

The 4-month RPT-MOX regimen is a treatment option for patients aged $\geq 12$ years with drug-susceptible pulmonary TB. Additional studies are needed to understand the pharmacokinetics and efficacy of the 4-month daily treatment regimen in patients for whom this regimen is not currently recommended, including young children, persons who are pregnant, patients with extrapulmonary TB, and patients with an HIV infection who are taking non-efavirenz-based antiretroviral therapy. Clinicians should carefully review a patient's clinical history, concurrent medications, social determinants of health, and risk 
factors for adverse drug reactions when making the decision to use this regimen.

Although neither RPT nor MOX has a labeling indication for a 4-month treatment of TB disease in the United States, RPT is recommended in U.S. guidelines as part of a preferred treatment regimen to prevent TB in persons with latent tuberculosis infection (8), and MOX is recommended as a drug for TB treatment (2). Available formulations of RPT, a key drug in the 4-month regimen, and of RIF, a key drug in standard 6-month TB treatment, have recently been found to contain low levels of nitrosamines.* More information about nitrosamines in these and other pharmaceuticals is available from the Food and Drug Administration (https://www.fda.gov/drugs/drug-safety-and-availability/ information-about-nitrosamine-impurities-medications).

Health care providers seeking clinical consultation should contact their state, tribal, local, and territorial health department TB programs (https://www.tbcontrollers.org/community/statecityterritory/) or the CDC-funded TB Centers of Excellence for Training, Education, and Medical Consultation (https://www.cdc.gov/tb/education/tb_coe/). CDC has information for health care providers and patients at https://www. cdc.gov/tb/topic/treatment/tbdisease.htm. CDC and other organizations will monitor the implementation of this interim guidance and update TB clinical guidelines as necessary.

\footnotetext{
*https://www.fda.gov/drugs/drug-safety-and-availability/fda-updates-and-pressannouncements-nitrosamines-rifampin-and-rifapentine
}

Corresponding author: Wendy Carr, ibt5@cdc.gov, 404-639-8583.

${ }^{1}$ Division of Tuberculosis Elimination, National Center for HIV, Viral Hepatitis, STD, and TB Prevention, CDC.

All authors have completed and submitted the International Committee of Medical Journal Editors form for disclosure of potential conflicts of interest. No potential conflicts of interest were disclosed.

\section{References}

1. Dorman SE, Nahid P, Kurbatova EV, et al.; AIDS Clinical Trials Group; Tuberculosis Trials Consortium. Four-month rifapentine regimens with or without moxifloxacin for tuberculosis. N Engl J Med 2021;384:1705-18. PMID:33951360 https://doi.org/10.1056/NEJMoa2033400

2. Nahid P, Dorman SE, Alipanah N, et al. Official American Thoracic Society/Centers for Disease Control and Prevention/Infectious Diseases Society of America clinical practice guidelines: treatment of drugsusceptible tuberculosis. Clin Infect Dis 2016;63:e147-95. PMID:27516382 https://doi.org/10.1093/cid/ciw376

3. Savic RM, Weiner M, MacKenzie WR, et al.; Tuberculosis Trials Consortium of the Centers for Disease Control and Prevention. Defining the optimal dose of rifapentine for pulmonary tuberculosis: exposureresponse relations from two phase II clinical trials. Clin Pharmacol Ther 2017;102:321-31. PMID:28124478 https://doi.org/10.1002/cpt.634

4. Rosenthal IM, Zhang M, Williams KN, et al. Daily dosing of rifapentine cures tuberculosis in three months or less in the murine model. PLoS Med 2007;4:e344. PMID:18092886 https://doi.org/10.1371/journal. pmed.0040344

5. Dorman SE, Savic RM, Goldberg S, et al.; Tuberculosis Trials Consortium. Daily rifapentine for treatment of pulmonary tuberculosis. A randomized, dose-ranging trial. Am J Respir Crit Care Med 2015;191:333-43. PMID:25489785 https://doi.org/10.1164/rccm.201410-1843OC

6. Rosenthal IM, Williams K, Tyagi S, et al. Weekly moxifloxacin and rifapentine is more active than the Denver regimen in murine tuberculosis. Am J Respir Crit Care Med 2005;172:1457-62. PMID:16141439 https:// doi.org/10.1164/rccm.200507-1072OC

7. Nahid P, Mase SR, Migliori GB, et al. Treatment of drug-resistant tuberculosis. An official ATS/CDC/ERS/IDSA clinical practice guideline. Am J Respir Crit Care Med 2019;200:e93-142. PMID:31729908 https:// doi.org/10.1164/rccm.201909-1874ST

8. Sterling TR, Njie G, Zenner D, et al. Guidelines for the treatment of latent tuberculosis infection: recommendations from the National Tuberculosis Controllers Association and CDC, 2020. MMWR Recomm Rep 2020;69(No. RR-1):1-11. PMID:32053584 https://doi.org/10.15585/ mmwr.rr6901a1 